\title{
Functional renormalization group for a large moiré unit cell
}

\author{
Lennart Klebl $\odot,{ }^{1}$ Dante M. Kennes $\odot,{ }^{1,2}$ and Carsten Honerkamp ${ }^{3}$ \\ ${ }^{1}$ Institute for Theory of Statistical Physics, RWTH Aachen University, and JARA Fundamentals of Future Information Technology, \\ 52062 Aachen, Germany \\ ${ }^{2}$ Max Planck Institute for the Structure and Dynamics of Matter, Center for Free Electron Laser Science, 22761 Hamburg, Germany \\ ${ }^{3}$ Institute for Theoretical Solid State Physics, RWTH Aachen University, and JARA Fundamentals of Future Information Technology, \\ 52062 Aachen, Germany
}

(Received 25 May 2020; accepted 14 July 2020; published 4 August 2020)

\begin{abstract}
Layers of two-dimensional materials arranged at a twist angle with respect to each other lead to enlarged unit cells with potentially strongly altered band structures, offering a new arena for novel and engineered many-body ground states. For the exploration of these, renormalization group methods are an appropriate, flexible tool that takes into account the mutual influence of competing tendencies. Here we show that, within reasonable, nontrivial approximations, the functional renormalization group known from simpler two-dimensional systems can be employed for the large-unit cell moiré superlattices with more than 10000 bands, remedying the need to employ ad hoc restrictions to effective low-energy theories of a few bands and/or effective continuum theories. This provides a description on the atomic scale, allowing one to absorb available ab initio information on the model parameters and therefore lending the analysis a more concrete quantitative character. For the case of twisted bilayer graphene models, we explore the leading ordering tendencies, depending on the band filling and the range of interactions. The results indicate a delicate balance between distinct magnetically ordered ground states, as well as the occurrence of a charge modulation within the moiré unit cell for sufficiently nonlocal repulsive interaction.
\end{abstract}

DOI: 10.1103/PhysRevB.102.085109

\section{INTRODUCTION}

In recent years, the field of two-dimensional materials has made major experimental and theoretical leaps, which led to many fascinating discoveries and have broadened our spectrum on available phases of matter in these highly controllable structures. Examples of these findings include superconducting [1,2] or magnetic [3] phases realized down to the monolayer limit and, related to that, the discovery of quantum anomalous Hall behavior in thin films [4-11], with potentially far-reaching technological applications in the realm of spintronics and quantum computing.

Recently, the twist angle between two sheets of material stacked atop each other was added as a further interesting research direction. In these twisted structures, it was shown that, by the emerging huge real space moiré supercells (tiny Brillouin zone), kinetic energy scales can be reduced drastically, giving rise to prominent interaction effects. In Refs. $[12,13]$, it was experimentally demonstrated that, using this route of control, two sheets of twisted bilayer graphene can be tuned to exhibit insulating as well as superconducting behavior. This exciting finding has spurred an enormous

Published by the American Physical Society under the terms of the Creative Commons Attribution 4.0 International license. Further distribution of this work must maintain attribution to the author(s) and the published article's title, journal citation, and DOI. Open access publication funded by the Max Planck Society. wave of experimental [14-23] as well as theoretical [24-36] research. Part of the excitement is founded in the fact that these two-dimensional systems can be controlled with relative ease using a backgate, strain, or the value of the twisting angle as a parameter. This allows one to access correlation regimes which might be otherwise difficult to access in a structure with a chemically straightforward composition (in this case graphene). From the theoretical side, a first approach is to concentrate on the correlation physics if we restrict ourselves to the low-energy bands near the Fermi surface. However, the validity of such an approach is difficult to assess. Often, when considering twisted van der Waals materials (like in the case of twisted bilayer graphene), there are no band gaps separating the lowest from higher energy bands and such a separation is unclear. Furthermore, topological arguments might obstruct the construction of such a simple low-energy theory [37]. In addition, a recent theoretical study shows that the interplay of correlations might be very subtle and fragile [38]. This calls for a different vantage point where, in contrast, we do not want to restrict ourselves to effective low-energy theories neglecting most of the many backfolded bands arising from the moiré supercell. When considering such a theory, we immediately face the problem that at small twist angles many thousand bands need to be kept. The sheer number of bands makes theoretical descriptions very cumbersome, especially when one tries to include interaction effects. Recent advances in this direction include Refs. [39-41], which treat correlation effects on the random-phase approximation (RPA) or meanfield level. 


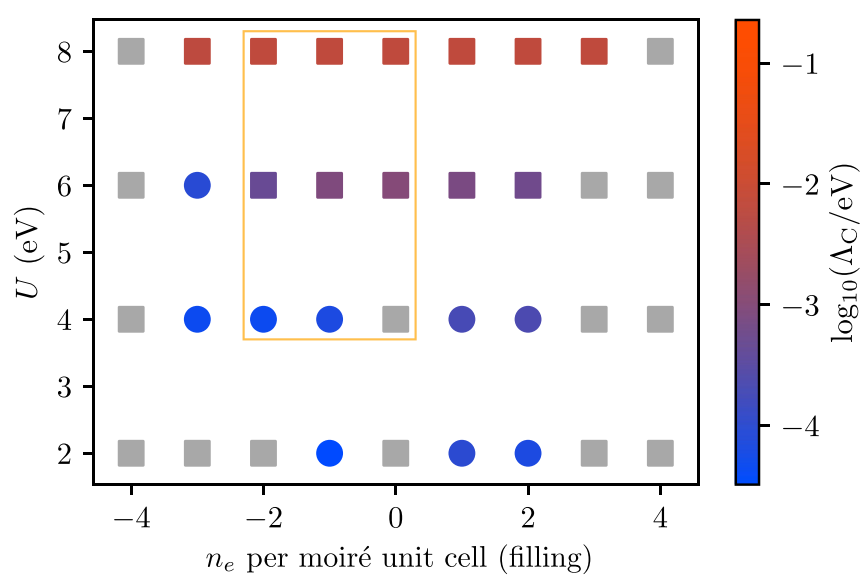

FIG. 1. Tentative phase diagram from the $\Gamma$-point IOBI fRG for twisted bilayer graphene at angle $1.05^{\circ}$ using a Hubbard interaction. The squares mark nodal antiferromagnetic order, the circles ferromagnetic order. For nondiverging flows, a gray square is shown. The color indicates the critical scale $\Lambda_{C}$ that roughly corresponds to the critical temperature of the phase transition. The orange rectangle shows the parameter region that simulations with longer ranged interactions have been carried out for (cf. Fig. 7). At strong interactions, the nodal antiferromagnetic phase is favored, whereas at smaller $U$, the system is susceptible to ferromagnetic ordering if its filling is close to the Van Hove singularities of the flat bands.

Here we want to add to this by establishing that a more sophisticated tool, the so-called functional renormalization group (fRG), can be applied to a Hamiltonian keeping the many bands in the moiré Brillouin zone, without reducing to effective low-energy theories. The fRG is a versatile method capable of describing a plethora of interacting electron systems [42-46]. Yet, solving the full fRG equations after a truncation at the two-particle vertex $\left(\Gamma^{(4)}\right)$ is in general numerically feasible only for systems with a few orbitals per unit cell, since the vertex function itself scales with the number of orbitals to the fourth power. In addition, the similarly rich dependence on momenta - or the unit cell positions when formulated in real space-remains a numerical challenge. Fortunately, for the latter dependence, reasonable simplifications can be formulated, with justifiable restrictions to short-ranged fermion bilinears $[43,47-50]$. Furthermore, at least for a larger set of questions, additional approximations simplifying the orbital dependence can be made [51,52]. Here we employ these two approximation steps to the fRG equations to keep the numerical effort low enough to treat systems with more than 10000 orbital sites per unit cell. We show that the treatment of twisted graphene bilayers close to the so-called magic angle using these approximations, resolving all individual carbon sites in the large moiré unit cell, yields similar results to what we know from our previous study using the RPA of the crossed particle-hole channel [41].

This paper extends such methodology to nonlocal interactions and the coupling to other channels beyond the RPA, and of course justifies the use of the RPA for the dominant instability a posteriori. We summarize the main results of this paper in the tentative phase diagram obtained with this method for a Hubbard interaction in Fig. 1. We find two magnetic orderings: First, antiferromagnetic order on the atomic scale

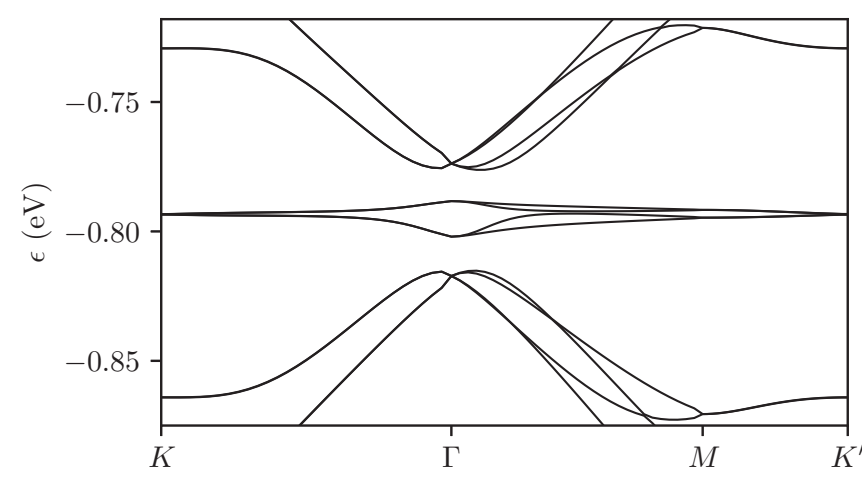

FIG. 2. Low-energy window of the noninteracting band structure of magic angle twisted bilayer graphene along the irreducible path in the moiré Brilluoin zone. There are four spin degenerate flat bands around charge neutrality at approximately $-0.79 \mathrm{eV}$.

with a sign change of the order parameter around the AA regions and, second, ferromagnetic order. The latter is only found at small interaction strengths and for fillings close to the Van Hove singularities of the material's flat bands at low critical scales. Nodal antiferromagnetism is present for all fillings that show an instability at larger couplings. With increasing nonlocal contributions to the interaction, the instability gets dominated by the repulsion among electrons outside the AA regions of the moire unit cell, indicating an interaction-induced charge redistribution.

The rest of this paper is structured as follows: We will shortly introduce the tight-binding model we use to describe magic angle twisted bilayer graphene in Sec. II. Thereafter, we discuss the method and approximations to it in Sec. III. Section IV shows the results of our simulations. Finally, we finish with some concluding remarks in Sec. V.

\section{MODEL}

We set up the moiré unit cell by constructing the superlattice vectors from lattice vectors of the honeycomb lattice of one single graphene sheet, $\boldsymbol{l}_{1}=(\sqrt{3} / 2,3 / 2,0)$ and $\boldsymbol{l}_{2}=$ $(\sqrt{3}, 0,0)$. The first superlattice vector can be written as $\boldsymbol{L}_{1}=$ $n \boldsymbol{l}_{1}+m \boldsymbol{l}_{2}$ with $n$ and $m$ integers defining the twist angle. The second superlattice vector $\boldsymbol{L}_{2}$ is rotated by $60^{\circ}$ with respect to $\boldsymbol{L}_{1}$. One of the layers is a honeycomb lattice with Bravais lattice vectors $\boldsymbol{l}_{1}$ and $\boldsymbol{l}_{2}$, the other one is shifted vertically and rotated by the twist angle $\theta=\arccos \frac{m^{2}+n^{2}+4 m n}{2\left(m^{2}+n^{2}+m n\right)}$ around the AA site. We implement corrugation effects by varying the interlayer distance in the supercell as described in Ref. [33]. Choosing $n=31, m=32$ sets $\theta=1.05^{\circ}$ and leads to 11908 sites in one supercell. The hopping parameters are taken from Ref. [53]. Once we have set up the Hamiltonian for one specific Bloch momentum $\boldsymbol{k}$ of magic angle twisted bilayer graphene, we diagonalize the matrix and obtain the spectrum $\epsilon_{b}(\boldsymbol{k})$ and the orbital makeup $u_{o b}(\boldsymbol{k})$. The low-energy part of the noninteracting band structure shows four (eight including spin) flat bands around charge neutrality (cf. Fig. 2). From the spectrum and orbital makeup, we can construct the free (Matsubara) Green's function as a matrix in orbital space:

$$
G_{o_{1} o_{2}}^{(0)}(k)=\sum_{b} \frac{u_{o_{1} b}(\boldsymbol{k}) u_{o_{2} b}^{*}(\boldsymbol{k})}{i k_{0}-\epsilon_{b}(\boldsymbol{k})+\mu} .
$$


As an electron-electron interaction, we employ the Ohno ansatz [54,55] with a sharp cutoff that allows us to treat increasingly longer ranged interactions. Its functional form in real space reads

$$
V_{O}(\boldsymbol{r})=\frac{U r_{O}}{\sqrt{r_{O}^{2}+\boldsymbol{r}^{2}}} \Theta\left(r_{c}-|\boldsymbol{r}|\right),
$$

where we introduced the Ohno radius $r_{O}$ and the cutoff radius $r_{c}$. For our simulations, we used $r_{O}=3 a$ ( $a$ is the graphene lattice constant), $r_{c} \in\{0.7 a, 1.1 a, 1.5 a\}$ (these correspond to nearest, next-nearest, and second-next-nearest-neighbor interactions in monolayer graphene) and the limit $r_{c} \rightarrow 0$, i.e., a Hubbard interaction. Note that there are more specific descriptions for the nonlocal interaction, also including the environmental screening [56].

For this qualitative fRG study, we concentrate on the simple form Eq. (2) and mainly study the dependence on $r_{c}$.

\section{METHOD}

We use the truncated unity approximation to the fRG equations which is described in more detail in Refs. [48,50], building essentially on earlier works, mainly Refs. [43,47]. The main idea of this scheme is to write the interaction vertex as a sum of the following three channels: direct particle-hole $(D)$, crossed particle-hole $(C)$, and particle-particle $(P)$ channel. Each part can be understood as an interaction between fermion bilinears of the corresponding types. The spatial structures of these bilinears can then be expanded in basis functions with specific symmetries in the moiré Brillouin zone. Even though the basis functions obey specific symmetry relations, they generally do not restrict the overall symmetry to specific sectors. This expansion is truncated in its length. As a simple but nontrivial approximation, we here consider only the in-cell contribution, i.e., the two fields in the fermion bilinears in pairing, charge, and spin channels are in the same unit cell. Given the large extent of the moiré unit cell, this already quite captures some spatial dependence and hence may be a tolerable approximation. In addition, we then employ a $\Gamma$ point approximation of the vertex function in the momentum argument of the three channels. This means that we ignore all momentum dependence of the interaction within the small moiré Brillouin zone. One can convince oneself that this provides a reasonable approximation as long as the interaction decays significantly in real space through the moiré unit cell. Due to the $\Gamma$-point approximation, we are sensitive with respect to order parameters that vary spatially within the moiré unit cell but that do not change when translated into other unit cells. Again, due to the large unit cell, this does not render the analysis trivial, but it excludes, e.g., density waves that enlarge the moiré unit cell. These can be investigated by allowing other wave vectors besides $\Gamma$, which we, however, postpone to later work. In addition, we neglect self-energy contributions as well as the frequency dependence of the vertex function and truncate the fRG equations at the four-point vertex $\Gamma^{(4)}$. Since we want to treat $S U$ (2) symmetric systems, we only need to consider the flow equations for the symmetrized vertex function $V^{\Lambda}$ where $\Lambda$ is the flow parameter. The three channels obey coupled differential equations in the flow parameter $\Lambda$ and carry orbital indices explicitly:

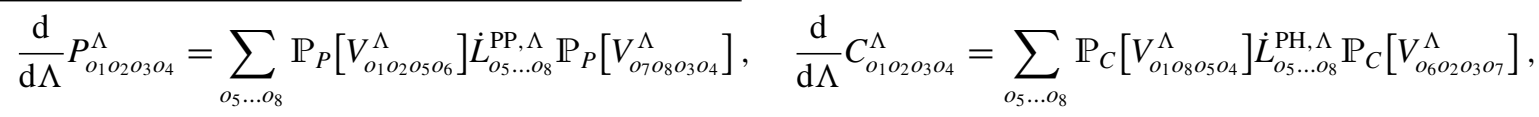

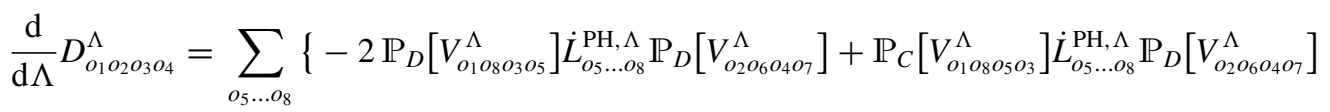

$$
\begin{aligned}
& \left.+\mathbb{P}_{D}\left[V_{O_{1} O_{8} O_{3} O_{5}}^{\Lambda}\right] \dot{L}_{0_{5} \ldots O_{8}}^{\mathrm{PH}, \Lambda} \mathbb{P}_{C}\left[V_{O_{2} O_{6} 0_{7} 0_{4}}^{\Lambda}\right]\right\},
\end{aligned}
$$

with the particle-particle and particle-hole loops:

$$
L_{0_{5} 0_{6} 0_{7} 0_{8}}^{\mathrm{PP}, \Lambda}=\frac{1}{\beta N} \sum_{k} G_{0_{5} 0_{7}}^{\Lambda}(k) G_{0_{6} 0_{8}}^{\Lambda}(-k), \quad L_{0_{5} 0_{6} 0_{7} 0_{8}}^{\mathrm{PH}, \Lambda}=\frac{1}{\beta N} \sum_{k} G_{0_{5} 0_{6}}^{\Lambda}(k) G_{0_{7} 0_{8}}^{\Lambda}(k) .
$$

The projection operators $\mathbb{P}$ reduce to unity without further approximations since they affect the momentum dependencies we disregarded. In the zero-temperature limit, the Matsubara sums in Eq. (4) become integrals. As we only need the differentiated loops $\mathrm{d} / \mathrm{d} \Lambda L^{\mathrm{PP} / \mathrm{PH}, \Lambda}$, it is in some cases possible to evaluate the integral analytically. For the sharp cutoff, we employ in our simulations, we use the Green's function $G_{o_{1} o_{2}}^{\Lambda}(k)=G_{o_{1} O_{2}}^{(0)}(k) \sqrt{\Theta\left(\left|k_{0}\right|-\Lambda\right)}$ and are able to trivially carry out the frequency integrals in the derivative of $L^{\mathrm{PP} / \mathrm{PH}, \Lambda}$ via the resulting delta function.

The approximation we make to be able to treat systems with a large number of orbitals is to only allow orbital bilinear interactions in each of the three interaction channels (IOBI approximation) [51]. This approximation is certainly valid as long as the interaction mainly consists of density-density type components. On the bare level, this is given as long as the Wannier orbitals used to construct the tight-binding model have a negligible overlap. But even beyond this simple situation, the approximation is capable of capturing the standard Kanamori representation of local interactions in terms of intra- and interorbital repulsions and Hund's rule parameters, as described in Ref. [51]. The IOBI approximation lowers the complexity of each channel of the vertex to only be number of orbitals to the power of two instead of four. This simplification reads

$$
\begin{aligned}
& P_{o_{1} O_{2} O_{3} O_{4}}^{\Lambda}=\delta_{O_{1} O_{2}} \delta_{O_{3} O_{4}} P_{O_{1} O_{3}}^{\Lambda}, \\
& C_{O_{1} O_{2} O_{3} O_{4}}^{\Lambda}=\delta_{O_{1} O_{4}} \delta_{O_{2} O_{3}} C_{O_{1} O_{3}}^{\Lambda}, \\
& D_{o_{1} O_{2} O_{3} O_{4}}^{\Lambda}=\delta_{O_{1} O_{3}} \delta_{O_{2} O_{4}} D_{o_{1} O_{3}}^{\Lambda} .
\end{aligned}
$$


The full vertex is composed by simply adding up the three channels:

$$
V_{o_{1} O_{2} O_{3} O_{4}}^{\Lambda}=P_{O_{1} O_{2} O_{3} O_{4}}^{\Lambda}+C_{O_{1} O_{2} O_{3} O_{4}}^{\Lambda}+D_{o_{1} O_{2} O_{3} O_{4}}^{\Lambda} .
$$

The initial interaction $V^{\infty}$ is of density-density type and can be written as a vertex function with components in the $D$ channel only. $V^{\Lambda}$ still is a fourth rank tensor in orbital space in the IOBI approximation, even though each channel is reduced to be a matrix in the orbital indices. The projections of the full vertex $V^{\Lambda}$ to a channel are now simply given by the restrictions of orbital bilinearity:

$$
\begin{aligned}
& \mathbb{P}_{P}\left[V_{O_{1} o_{2} o_{3} o_{4}}^{\Lambda}\right]=\delta_{O_{1} o_{2}} \delta_{O_{3} o_{4}} V_{o_{1} o_{2} o_{3} o_{4}}^{\Lambda}, \\
& \mathbb{P}_{C}\left[V_{o_{1} o_{2} o_{3} o_{4}}^{\Lambda}\right]=\delta_{O_{1} o_{4}} \delta_{o_{2} O_{3}} V_{o_{1} o_{2} o_{3} o_{4}}^{\Lambda}, \\
& \mathbb{P}_{D}\left[V_{o_{1} o_{2} o_{3} o_{4}}^{\Lambda}\right]=\delta_{O_{1} o_{3}} \delta_{o_{2} o_{4}} V_{o_{1} o_{2} o_{3} o_{4}}^{\Lambda} .
\end{aligned}
$$

Insertion of the channel projections into Eq. (3) yields the flow equations in the static IOBI $\Gamma$-point approximation:

$$
\begin{aligned}
\frac{\mathrm{d}}{\mathrm{d} \Lambda} \hat{P}^{\Lambda}= & \hat{V}^{\mathbb{P}_{P}, \Lambda} \dot{\hat{L}}^{\mathrm{PP}, \Lambda} \hat{V}^{\mathbb{P}_{P}, \Lambda}, \\
\frac{\mathrm{d}}{\mathrm{d} \Lambda} \hat{C}^{\Lambda}= & \hat{V}^{\mathbb{P}_{C}, \Lambda} \dot{\hat{L}}^{\mathrm{PH}, \Lambda} \hat{V}^{\mathbb{P}_{C}, \Lambda}, \\
\frac{\mathrm{d}}{\mathrm{d} \Lambda} \hat{D}^{\Lambda}= & -2 \hat{V}^{\mathbb{P}_{D}, \Lambda} \dot{\hat{L}}^{\mathrm{PH}, \Lambda} \hat{V}^{\mathbb{P}_{D}, \Lambda}+\hat{V}^{\mathbb{P}_{D}, \Lambda} \dot{\hat{L}}^{\mathrm{PH}, \Lambda} \hat{V}^{\mathbb{P}_{C}, \Lambda} \\
& +\hat{V}^{\mathbb{P}_{C}, \Lambda} \dot{\hat{L}}^{\mathrm{PH}, \Lambda} \hat{V}^{\mathbb{P}_{D}, \Lambda} .
\end{aligned}
$$

The quantities are all matrices and connected by matrix products. The projected vertex function's matrices read

$$
\begin{aligned}
& \hat{V}^{\mathbb{P}_{P}, \Lambda}=\hat{P}^{\Lambda}+\operatorname{diag}\left(\hat{C}^{\Lambda}\right)+\operatorname{diag}\left(\hat{D}^{\Lambda}\right), \\
& \hat{V}^{\mathbb{P}_{C}, \Lambda}=\operatorname{diag}\left(\hat{P}^{\Lambda}\right)+\hat{C}^{\Lambda}+\operatorname{diag}\left(\hat{D}^{\Lambda}\right), \\
& \hat{V}^{\mathbb{P}_{D}, \Lambda}=\operatorname{diag}\left(\hat{P}^{\Lambda}\right)+\operatorname{diag}\left(\hat{C}^{\Lambda}\right)+\hat{D}^{\Lambda} .
\end{aligned}
$$

By the IOBI approximation, the loops are constrained to be matrices as well and take the following form:

$$
\begin{aligned}
\hat{L}^{\mathrm{PP}, \Lambda} & =\int \frac{\mathrm{d} k_{0}}{2 \pi} \frac{1}{N} \sum_{k} \hat{G}^{\Lambda}(k) \circ \hat{G}^{\Lambda}(-k), \\
\hat{L}^{\mathrm{PH}, \Lambda} & =\int \frac{\mathrm{d} k_{0}}{2 \pi} \frac{1}{N} \sum_{k} \hat{G}^{\Lambda}(k) \circ \hat{G}^{\Lambda}(k),
\end{aligned}
$$

where the operation $\hat{A} \circ \hat{B}$ is an elementwise matrix product of matrices $\hat{A}$ and $\hat{B}$.

Starting from a value $\Lambda_{\text {ini }}=10 \mathrm{eV}$ for the frequency cutoff, we integrate the flow to $\Lambda_{0}=0$. The value of the frequency step $\mathrm{d} \Lambda$ as a function of $\Lambda$ is adaptively chosen in the window given by two envelope functions as shown in Fig. 3. Quite generally, the fRG flow leads to strong coupling, i.e., a rapid growth of certain components of the flowing interaction. We draw physical conclusions from this by calculating the maximum eigenvalue of each interaction channel for each step for the intraorbital bilinear matrices $\hat{P}^{\Lambda}, \hat{C}^{\Lambda}$, and $\hat{D}^{\Lambda}$. If one of the eigenvalues surpasses a critical value (which we set to $10^{3} \mathrm{eV}$ ), we stop the flow and do a full eigendecomposition of the three channel matrices. The eigenvector corresponding to the maximum eigenvalue indicates the orbital character of the order parameter associated to the divergence. An example of several fRG flows at fixed filling and interaction strength

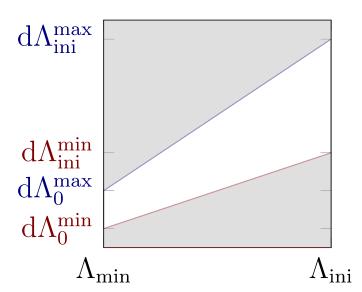

FIG. 3. Sketch of envelope functions to choose $\mathrm{d} \Lambda$ as a function of $\Lambda$ for the integration of the fRG flow. The gray area is inaccessible, $\mathrm{d} \Lambda$ has to be between the red and blue functions in the white area. In our simulations, we restricted $\mathrm{d} \Lambda$ by two power-law boundaries with exponent 1.1 to maximize numerical efficiency.

for different interaction cutoffs is shown in Fig. 4. Including nearest-neighbor interaction leads to a divergence in the $D$ channel compared to the magnetic instability in the $C$ channel found for on-site and longer ranged interactions.

\section{RESULTS}

The three main types of ordering found as runaway flows in this paper are shown in terms of their leading eigenvectors in Fig. 5: (a) ferromagnetic order throughout the unit cell with some residual antiferromagnetism on the carbon-carbon-bond scale in the $\mathrm{AB}$ regions, (b) nodal antiferromagnetic order with a sign change of the antiferromagnetic order parameter on the atomic scale around the AA regions, and (c) chargemodulated states that form a honeycomb lattice of the $\mathrm{AB}$ and $\mathrm{BA}$ regions. This last instability is characterized by a leading eigenvector in the density channel. It should be analyzed in its pure form for $r_{c}=1.5 a$ and $U=8 \mathrm{eV}$ where the sign change in the eigenvector occurring for smaller $r_{c}$ and $U$ has disappeared. Then the charge modulation (CM) arises from the leading eigenvector that has the largest weight in the $\mathrm{AB}$ regions. This represents a strong growth of the effective

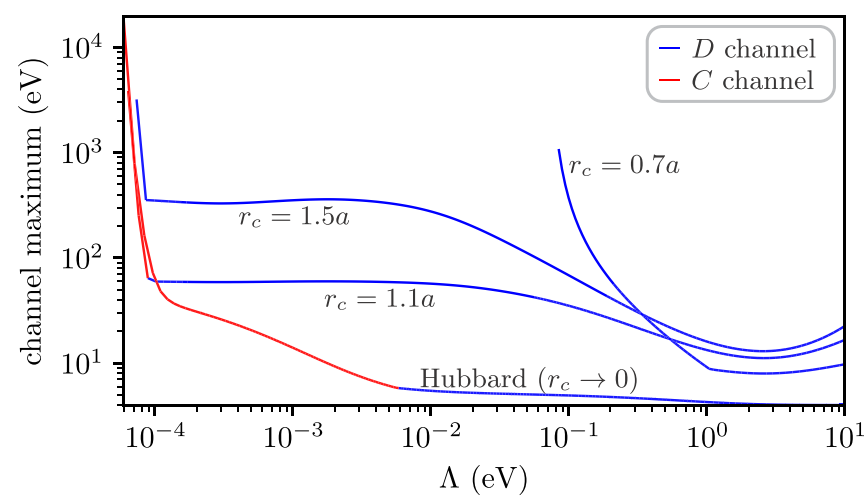

FIG. 4. fRG flow of the maximal channel of the interactions with different interaction cutoffs $r_{c}$ and on-site Hubbard interaction $\left(r_{c} \rightarrow 0\right)$. The lines' colors represent whether the vertex function's maximum occurs in the $C$ channel (red) or the $D$ channel (blue). The system is doped to -1 electron per moiré unit cell with an onsite interaction strength $U=4 \mathrm{eV}$. For a cutoff at nearest-neighbor interaction $\left(r_{c}=0.7\right)$, a $D$ channel instability is favored whereas for the other interactions, it has a $C$ channel instability at much lower critical value $\Lambda_{C}$. 
(a)

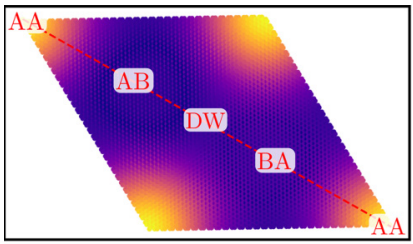

(b)

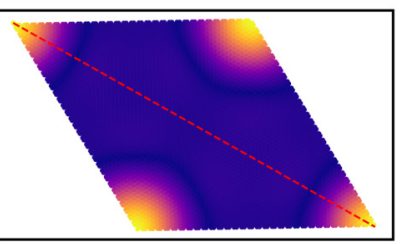

(c)

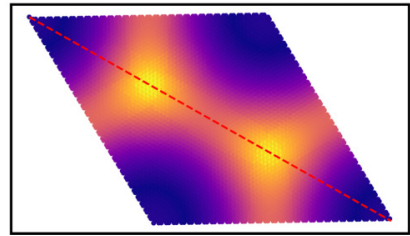

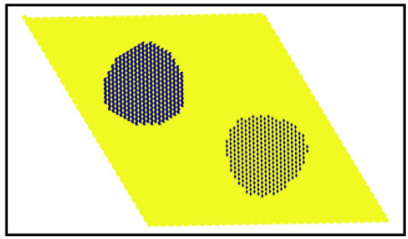
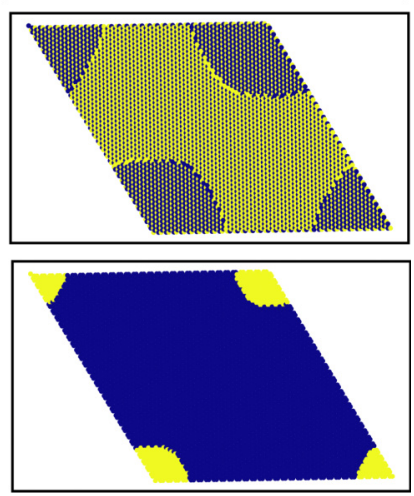

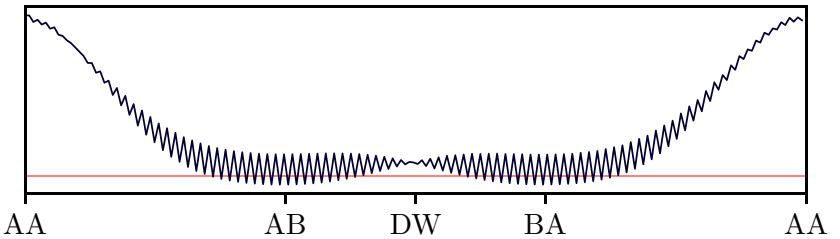

AA
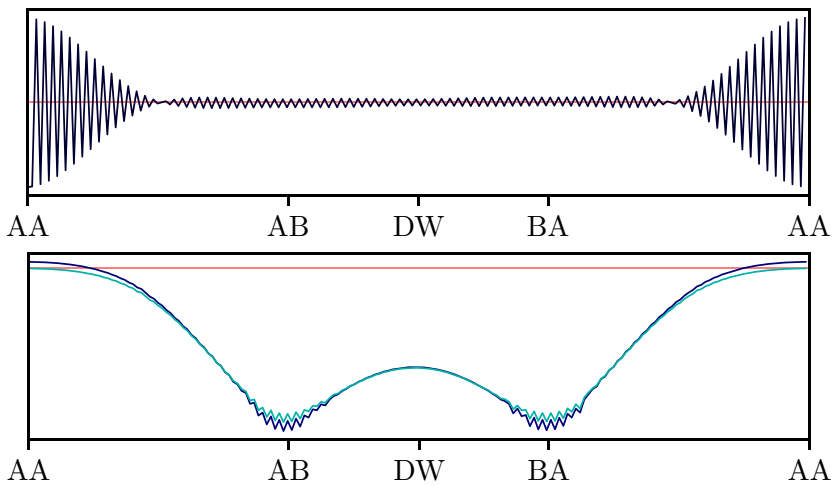

FIG. 5. The three different orderings found in this paper represented by the eigenvectors of the leading channel at the end of the fRG flow. (a) Ferromagnetic ordering, $C$ channel, Hubbard interaction $\left(r_{c} \rightarrow 0\right),-1$ electron per moiré unit cell, $U=4 \mathrm{eV}$, (b) nodal antiferromagnetic ordering, $C$ channel, Hubbard interaction $\left(r_{c} \rightarrow 0\right)$, charge neutrality, $U=6 \mathrm{eV}$, (c) charge-modulation ordering eigenvector, $D$ channel, Ohno interaction with $r_{c}=1.5 a,-1$ electron per moiré unit cell, $U=6 \mathrm{eV}$. The left column is the absolute value of the corresponding eigenvector for the top layer (blue corresponds to zero, yellow to the maximum), the center column is the sign (blue: minus, yellow: plus) and the right column is the value along the dashed red line through the rhomb-shaped unit cell. In the right panel of (c), the cyan line indicates another realization of $D$ channel charge-modulation ordering where no sign change in the eigenvector is present. It corresponds to the on-site interaction strength $U=8 \mathrm{eV}$.

(a)

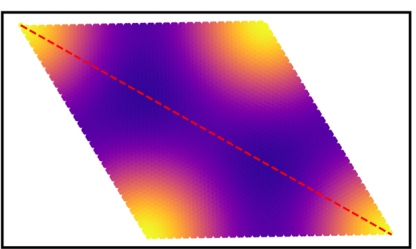

(b)
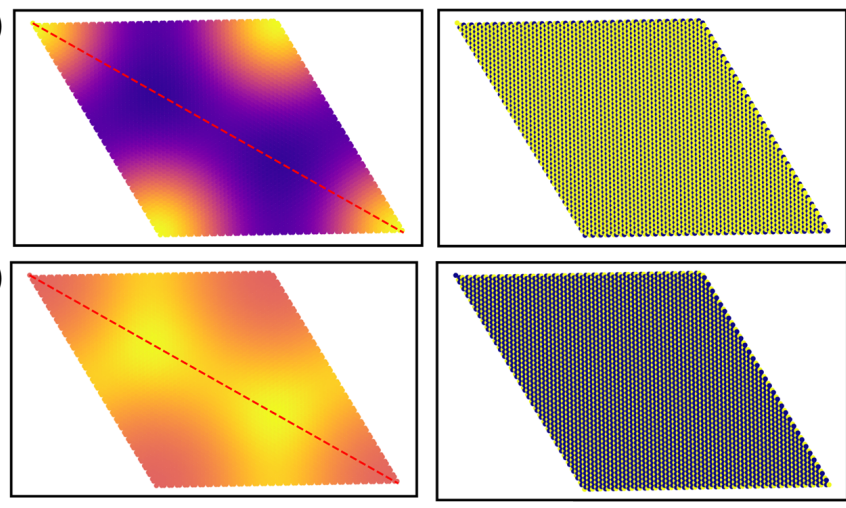

$(\mathrm{c} 1)$

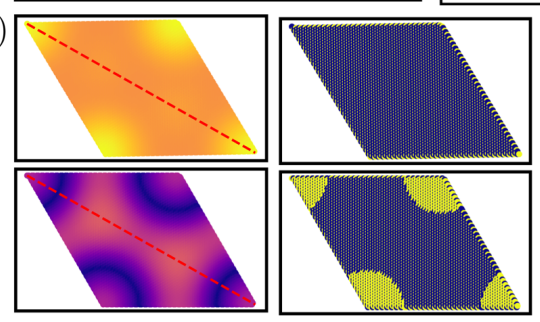

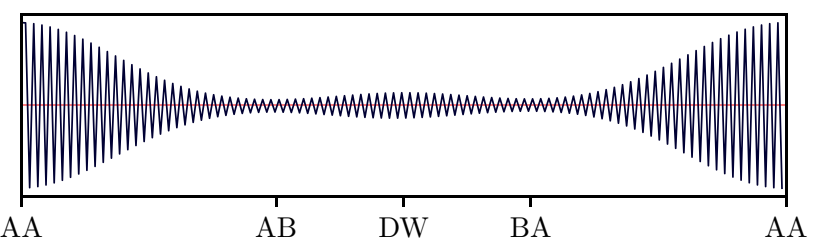

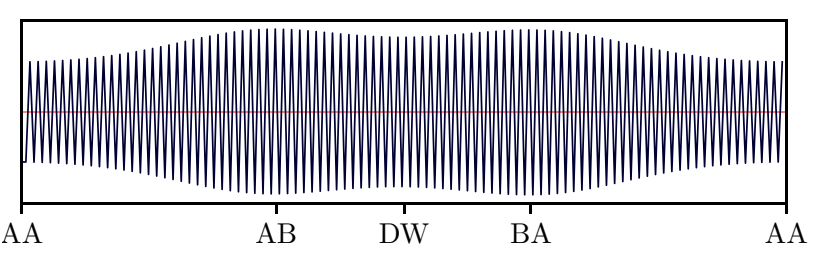

(c2)

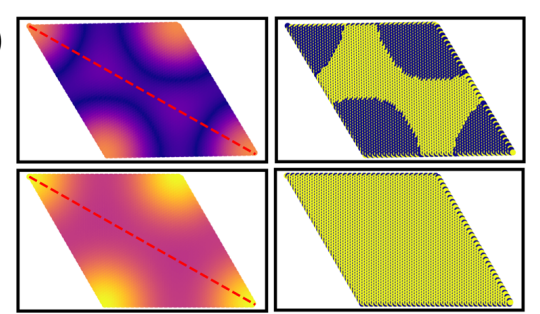

FIG. 6. Charge-density wave (CDW) orderings found for a cutoff at nearest-neighbor interaction $\left(r_{c}=0.7 a\right)$. (a) $U=4 \mathrm{eV}$, (b) $U=8 \mathrm{eV}$, and (c1), (c2) $U=6 \mathrm{eV}$. The leading eigenvector of the $D$ channel is shown. As in Fig. 5, the left column is the absolute value of the eigenvector for the top layer (blue corresponds to zero, yellow to the maximum), the center column the sign (blue: minus, yellow: plus), and the right column the value along the dashed red line through the rhomb-shaped unit cell. (c1) shows the leading and (c2) the subleading eigenvector for both layers. Note that the sign change of the staggering around the AA sites is in the lower layer for the leading eigenvector and in the upper layer for the subleading eigenvector. 


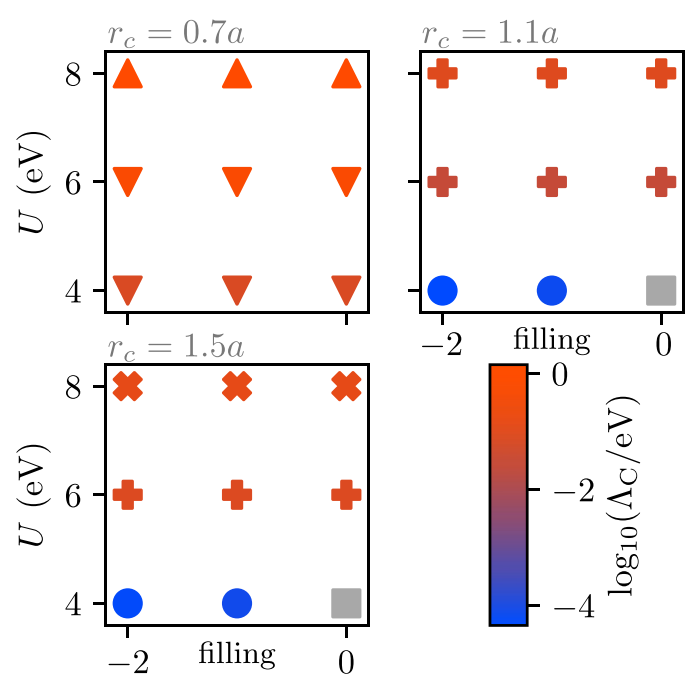

FIG. 7. Dependence of the phase diagram of magic angle twisted bilayer graphene on the range of the interaction $r_{c}$. Squares mark nodal antiferromagnetic order, circles ferromagnetic order, plus symbols CM states without sign change and crosses CM states with sign change. For $r_{c}=0.7 a$, a CDW state (on the carbon-carbon-bond scale) with slight amplitude modulation on the moiré scale is found. These states are marked by triangles. Gray squares indicate that the vertex did not diverge before reaching $\Lambda=0$.

electronic repulsion in those regions, which should then push the charges away from there into the AA regions.

\section{A. Hubbard interaction $\left(r_{c} \rightarrow 0\right)$}

For on-site interaction, we find instabilities of magnetic type [cf. Figs. 5(a) and 5(b)] that agree with the types of ordering we found in our previous RPA study for the magnetic susceptibility due to a pure on-site interaction [41]. Additionally, the values of $\Lambda_{C}$ are similar in their order of magnitude to the critical temperatures found in RPA. The phase diagram obtained from fRG using a Hubbard interaction $\left(r_{c} \rightarrow 0\right)$ is shown in Fig. 1.

Ferromagnetism is present for fillings close to the Van Hove singularities of the flat bands and small interaction strengths. Increasing $U$ or doping away from the Van Hove singularities leads to nodal antiferromagnetism.

\section{B. Longer ranged interactions}

For longer ranged interactions, we find that the system is susceptible to a $\mathrm{CM}$ or charge-density wave (CDW) instability. For the fillings and interaction strengths indicated by the orange square in Fig. 1, we carried out the simulations with longer range interactions. The results are summarized in a series of additional tentative phase diagrams in Fig. 7. We observe that for the cases where magnetism is found $\left(r_{c}>\right.$ $0.7 a, U=4 \mathrm{eV}$ ), the critical scale $\Lambda_{C}$ is almost independent of whether the interaction is longer ranged or of Hubbard type $\left(r_{c} \rightarrow 0\right)$. CM ordering becomes relevant for larger $U$ $\left(r_{c}>0.7\right)$. While the quantitative location of the AF-to-CM transition has to be checked in more elaborate numerical studies, our current finding indicates a high sensitivity of the interacting system with respect to nonlocal interaction, as also

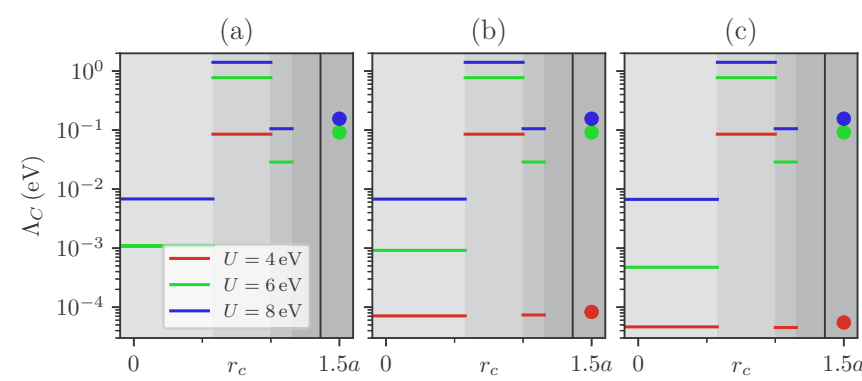

FIG. 8. Dependence of $\Lambda_{C}$ on range of the interaction $r_{c}$. (a) shows the results for charge neutrality, (b) for doping of -1 electron per moiré unit cell, and (c) for -2 electrons per moiré unit cell. The CM ordering at $U>4 \mathrm{eV}$ and $r_{c} \geqslant a$ consistently shows an increase of $\Lambda_{C}$ for all fillings. The differently shaded regions indicate the regions where the interaction includes the first-, second-, third-, and fourth-nearest neighbor. Beyond the black vertical line, interlayer couplings are present in the initial interaction.

stated in Ref. [38]. Additionally, the critical scale takes much larger values for both the $\mathrm{CM}$ and $\mathrm{CDW}$ instabilities (see Fig. 8).

Including nearest-neighbor interactions $\left(r_{c}=0.7 a\right)$ leads to CDW states as dominant instability. In the context of inheriting the instabilities from AA- and AB-stacked graphene bilayers, CDW states with opposite charge density modulation on $\mathrm{A}$ and $\mathrm{B}$ carbon sublattices are expected at longer ranged interactions [57,58]. Figure 6 shows the CDW states on the carbon-carbon-bond scale with slight modulations on the moiré scale. The case $U=6 \mathrm{eV}$ shows two almost degenerate orderings with (approximate) layer degeneracy.

As soon as next-nearest-neighbor interactions are included, $\left(r_{c}>0.7\right)$ the $\mathrm{CDW}$ states for $U \geqslant 6 \mathrm{eV}$ get replaced by $\mathrm{CM}$ states that feature a variation of the effective densitydensity interaction on the much longer moiré scale and no staggering on the carbon-carbon-bond scale [cf. Fig. 5(c)]. This interaction is expected to drive a charge transfer within the unit cell. As the interaction is stronger in the $\mathrm{AB}$ regions, as visible from the absolute magnitude of the eigenvector in Fig. 5(c), the charge density should get lowered there.

To support the validity of the fRG method used in this study, we set up simulations of single-layer graphene in a supercell of approximately 1000 sites and could reproduce the results for varying nearest- and next-nearest-neighbor interaction strengths found in fRG studies with high wavevector resolution [59] (not shown). This earlier study also showed a variation of the CDW wave vector toward the $\Gamma$ point for increasingly nonlocal interactions, consistent with the findings in the moiré systems here.

\section{CONCLUSION}

We use the fRG to describe interactions and their ordering tendencies in large moiré unit cells. The results for twisted bilayer graphene at magic angles with pure on-site interactions agree with our previous RPA study. Using the fRG allows us to include long-range interactions in addition. Here, we find charge modulated and CDW states besides magnetic orderings and investigate the competition between the different ordering tendencies. 
Until now, only s-wave correlations are allowed by our approximations. Even in this approximation scheme, we are able to show how different order parameters emerge, some of which are inherited from nontwisted graphene bilayer systems (antiferromagnetism on the atomic scale and CDW on the atomic scale). Additionally, the longer ranged Ohno interaction that is accessible in fRG leads to a competition between direct- and crossed-particle-hole channels, i.e., charge and magnetic fluctuations. As a strong competitor of the magnetic order we find an intra-moiré-cell charge-modulated state for which the Coulomb repulsion in the $\mathrm{AB}$ regions between the AA spots flows to strong coupling. To the best of our knowledge, this instability has not been discussed before and deserves further studies. In particular, one should understand whether this state can help explain the phenomenology of twisted-bilayer graphene systems, e.g., by depleting the lowenergy density of states between the AA regions even further and therefore localizing electrons in nearly isolated AA islands. Furthermore, it might well be that, once the charge redistribution has been accounted for, e.g., by self-energy terms, the previous magnetic instabilities become relevant again beyond the parameter range found in this work.
The main goal of this paper was to demonstrate that one can set up fRG calculations within useful approximations even for such systems with $\mathcal{O}\left(10^{4}\right)$ bands. We plan to extend the method to allow for more quantitative comparisons and also to treat other pairing bilinears in the $P$ channel and momentum dependencies in the other channels. This will allow us to study, e.g., unconventional superconductivity and other bond ordering phenomena on the moiré scale in the IOBI fRG for systems like magic angle twisted bilayer graphene.

\section{ACKNOWLEDGMENTS}

The German Science Foundation (DFG) is acknowledged for support through RTG 1995 and under Germany's Excellence Strategy - Cluster of Excellence Matter and Light for Quantum Computing (ML4Q) EXC 2004/1 - 390534769. Support by the Max Planck Institute-New York City Center for Non-Equilibrium Quantum Phenomena is acknowledged. Most simulations were performed with computing resources granted by RWTH Aachen University under Project No. rwth0496. In addition, the authors also gratefully acknowledge the computing time granted through JARA on the supercomputer JURECA at Forschungszentrum Jülich [60].
[1] X. Xi, L. Zhao, Z. Wang, H. Berger, L. Forró, J. Shan, and K. F. Mak, Strongly enhanced charge-density-wave order in monolayer $\mathrm{NbSe}_{2}$, Nat. Nanotechnol. 10, 765 (2015).

[2] A. Benyamini, E. J. Telford, D. M. Kennes, D. Wang, A. Williams, K. Watanabe, T. Taniguchi, D. Shahar, J. Hone, C. R. Dean, A. J. Millis, and A. N. Pasupathy, Fragility of the dissipationless state in clean two-dimensional superconductors, Nat. Phys. 15, 947 (2019).

[3] J. Stajic, A detailed look into 2D magnetism, Science 364, 966 (2019).

[4] C.-Z. Chang, J. Zhang, X. Feng, J. Shen, Z. Zhang, M. Guo, K. Li, Y. Ou, P. Wei, L.-L. Wang, Z.-Q. Ji, Y. Feng, S. Ji, X. Chen, J. Jia, X. Dai, Z. Fang, S.-C. Zhang, K. He, Y. Wang, L. Lu, X.-C. Ma, and Q.-K. Xue, Experimental observation of the quantum anomalous Hall effect in a magnetic topological insulator, Science 340, 167 (2013).

[5] X. Kou, S.-T. Guo, Y. Fan, L. Pan, M. Lang, Y. Jiang, Q. Shao, T. Nie, K. Murata, J. Tang et al., Scale-Invariant Quantum Anomalous Hall Effect in Magnetic Topological Insulators Beyond the Two-Dimensional Limit, Phys. Rev. Lett. 113, 137201 (2014).

[6] J. Checkelsky, R. Yoshimi, A. Tsukazaki, K. Takahashi, Y. Kozuka, J. Falson, M. Kawasaki, and Y. Tokura, Trajectory of the anomalous Hall effect towards the quantized state in a ferromagnetic topological insulator, Nat. Phys. 10, 731 (2014).

[7] A. J. Bestwick, E. J. Fox, X. Kou, L. Pan, K. L. Wang, and D. Goldhaber-Gordon, Precise Quantization of the Anomalous Hall Effect Near Zero Magnetic Field, Phys. Rev. Lett. 114, 187201 (2015).

[8] C.-Z. Chang, W. Zhao, D. Y. Kim, H. Zhang, B. A. Assaf, D. Heiman, S.-C. Zhang, C. Liu, M. H. Chan, and J. S. Moodera, High-precision realization of robust quantum anomalous Hall state in a hard ferromagnetic topological insulator, Nat. Mater. 14, 473 (2015).
[9] A. Kandala, A. Richardella, S. Kempinger, C.-X. Liu, and N. Samarth, Giant anisotropic magnetoresistance in a quantum anomalous Hall insulator, Nat. Commun. 6, 7434 (2015).

[10] X. Kou, L. Pan, J. Wang, Y. Fan, E. S. Choi, W.-L. Lee, T. Nie, K. Murata, Q. Shao, S.-C. Zhang et al., Metal-to-insulator switching in quantum anomalous Hall states, Nat. Commun. 6, 8474 (2015).

[11] Y. Feng, X. Feng, Y. Ou, J. Wang, C. Liu, L. Zhang, D. Zhao, G. Jiang, S.-C. Zhang, K. He, X. Ma, Q.-K. Xue, and Y. Wang, Observation of the Zero Hall Plateau in a Quantum Anomalous Hall Insulator, Phys. Rev. Lett. 115, 126801 (2015).

[12] Y. Cao, V. Fatemi, S. Fang, K. Watanabe, T. Taniguchi, E. Kaxiras, and P. Jarillo-Herrero, Unconventional superconductivity in magic-angle graphene superlattices, Nature 556, 43 (2018).

[13] Y. Cao, V. Fatemi, A. Demir, S. Fang, S. L. Tomarken, J. Y. Luo, J. D. Sanchez-Yamagishi, K. Watanabe, T. Taniguchi, E. Kaxiras et al., Correlated insulator behavior at half-filling in magic-angle graphene superlattices, Nature 556, 80 (2018).

[14] M. Yankowitz, S. Chen, H. Polshyn, Y. Zhang, K. Watanabe, T. Taniguchi, D. Graf, A. F. Young, and C. R. Dean, Tuning superconductivity in twisted bilayer graphene, Science 363, 1059 (2019).

[15] A. Kerelsky, L. J. McGilly, D. M. Kennes, L. Xian, M. Yankowitz, S. Chen, K. Watanabe, T. Taniguchi, J. Hone, C. Dean et al., Maximized electron interactions at the magic angle in twisted bilayer graphene, Nature 572, 95 (2019).

[16] Y. Choi, J. Kemmer, Y. Peng, A. Thomson, H. Arora, R. Polski, Y. Zhang, H. Ren, J. Alicea, G. Refael et al., Electronic correlations in twisted bilayer graphene near the magic angle, Nat. Phys. 15, 1174 (2019).

[17] X. Lu, P. Stepanov, W. Yang, M. Xie, M. A. Aamir, I. Das, C. Urgell, K. Watanabe, T. Taniguchi, G. Zhang et al., 
Superconductors, orbital magnets and correlated states in magic-angle bilayer graphene, Nature 574, 653 (2019).

[18] C. Shen, Y. Chu, Q. Wu, N. Li, S. Wang, Y. Zhao, J. Tang, J. Liu, J. Tian, K. Watanabe et al., Correlated states in twisted double bilayer graphene, Nat. Phys. 16, 520 (2020).

[19] X. Liu, Z. Hao, E. Khalaf, J. Y. Lee, Y. Ronen, H. Yoo, D. H. Najafabadi, K. Watanabe, T. Taniguchi, A. Vishwanath, and P. Kim, Tunable spin-polarized correlated states in twisted double bilayer graphene, Nature 583, 221 (2020).

[20] Y. Cao, D. Rodan-Legrain, O. Rubies-Bigorda, J. M. Park, K. Watanabe, T. Taniguchi, and P. Jarillo-Herrero, Tunable correlated states and spin-polarized phases in twisted bilayer-bilayer graphene, Nature 583, 215 (2020).

[21] Y. Jiang, X. Lai, K. Watanabe, T. Taniguchi, K. Haule, J. Mao, and $\mathrm{E}$. Y. Andrei, Charge order and broken rotational symmetry in magic-angle twisted bilayer graphene, Nature 573, 91 (2019).

[22] L. Wang, E.-M. Shih, A. Ghiotto, L. Xian, D. A. Rhodes, C. Tan, M. Claassen, D. M. Kennes, Y. Bai, B. Kim, K. Watanabe, T. Taniguchi, X. Zhu, J. Hone, A. Rubio, A. Pasupathy, and C. R. Dean, Magic continuum in twisted bilayer $\mathrm{WSe}_{2}$, arXiv:1910.12147 [cond-mat.mes-hall].

[23] A. Kerelsky, C. Rubio-Verdú, L. Xian, D. M. Kennes, D. Halbertal, N. Finney, L. Song, S. Turkel, L. Wang, K. Watanabe, T. Taniguchi, J. Hone, C. Dean, D. Basov, A. Rubio, and A. N. Pasupathy, Moiré-less correlations in ABCA graphene, arXiv:1911.00007 [cond-mat.mes-hall].

[24] G. Trambly de Laissardière, D. Mayou, and L. Magaud, Localization of Dirac electrons in rotated graphene bilayers, Nano Lett. 10, 804 (2010).

[25] G. Li, A. Luican, J. L. Dos Santos, A. C. Neto, A. Reina, J. Kong, and E. Andrei, Observation of Van Hove singularities in twisted graphene layers, Nat. Phys. 6, 109 (2010).

[26] R. Bistritzer and A. H. MacDonald, Moiré bands in twisted double-layer graphene, Proc. Natl. Acad. Sci. 108, 12233 (2011)

[27] J. M. B. Lopes dos Santos, N. M. R. Peres, and A. H. Castro Neto, Continuum model of the twisted graphene bilayer, Phys. Rev. B 86, 155449 (2012).

[28] I. Brihuega, P. Mallet, H. González-Herrero, G. Trambly de Laissardière, M. M. Ugeda, L. Magaud, J. M. GómezRodríguez, F. Ynduráin, and J.-Y. Veuillen, Unraveling the Intrinsic and Robust Nature of Van Hove Singularities in Twisted Bilayer Graphene by Scanning Tunneling Microscopy and Theoretical Analysis, Phys. Rev. Lett. 109, 196802 (2012).

[29] Y. Kim, P. Herlinger, P. Moon, M. Koshino, T. Taniguchi, K. Watanabe, and J. H. Smet, Charge inversion and topological phase transition at a twist angle induced Van Hove singularity of bilayer graphene, Nano Lett. 16, 5053 (2016).

[30] H. C. Po, L. Zou, A. Vishwanath, and T. Senthil, Origin of Mott Insulating Behavior and Superconductivity in Twisted Bilayer Graphene, Phys. Rev. X 8, 031089 (2018).

[31] B. Roy and V. Juričić, Unconventional superconductivity in nearly flat bands in twisted bilayer graphene, Phys. Rev. B 99, 121407(R) (2019).

[32] J. Kang and O. Vafek, Symmetry, Maximally Localized Wannier States, and a Low-Energy Model for Twisted Bilayer Graphene Narrow Bands, Phys. Rev. X 8, 031088 (2018).

[33] M. Koshino, N. F. Q. Yuan, T. Koretsune, M. Ochi, K. Kuroki, and $\mathrm{L}$. Fu, Maximally Localized Wannier Orbitals and the
Extended Hubbard Model for Twisted Bilayer Graphene, Phys. Rev. X 8, 031087 (2018).

[34] L. Xian, D. M. Kennes, N. Tancogne-Dejean, M. Altarelli, and A. Rubio, Multiflat bands and strong correlations in twisted bilayer boron nitride: Doping-induced correlated insulator and superconductor, Nano Lett. 19, 4934 (2019).

[35] P. Lucignano, D. Alfè, V. Cataudella, D. Ninno, and G. Cantele, Crucial role of atomic corrugation on the flat bands and energy gaps of twisted bilayer graphene at the magic angle $\theta \sim 1.08^{\circ}$, Phys. Rev. B 99, 195419 (2019).

[36] D. M. Kennes, L. Xian, M. Claassen, and A. Rubio, Onedimensional flat bands in twisted bilayer germanium selenide, Nat. Commun. 11, 1124 (2020).

[37] H. C. Po, L. Zou, T. Senthil, and A. Vishwanath, Faithful tightbinding models and fragile topology of magic-angle bilayer graphene, Phys. Rev. B 99, 195455 (2019).

[38] R. E. Throckmorton and S. D. Sarma, Spontaneous symmetry breaking in a honeycomb lattice subject to a periodic potential, Phys. Rev. Research 2, 023225 (2020).

[39] L. A. Gonzalez-Arraga, J. L. Lado, F. Guinea, and P. SanJose, Electrically Controllable Magnetism in Twisted Bilayer Graphene, Phys. Rev. Lett. 119, 107201 (2017).

[40] M. Xie and A. H. MacDonald, Nature of the Correlated Insulator States in Twisted Bilayer Graphene, Phys. Rev. Lett. 124, 097601 (2020).

[41] L. Klebl and C. Honerkamp, Inherited and flatband-induced ordering in twisted graphene bilayers, Phys. Rev. B 100, 155145 (2019).

[42] W. Metzner, M. Salmhofer, C. Honerkamp, V. Meden, and K. Schönhammer, Functional renormalization group approach to correlated fermion systems, Rev. Mod. Phys. 84, 299 (2012).

[43] W.-S. Wang, Y.-Y. Xiang, Q.-H. Wang, F. Wang, F. Yang, and D.-H. Lee, Functional renormalization group and variational Monte Carlo studies of the electronic instabilities in graphene near $\frac{1}{4}$ doping, Phys. Rev. B 85, 035414 (2012).

[44] C. Platt, W. Hanke, and R. Thomale, Functional renormalization group for multi-orbital Fermi surface instabilities, Adv. Phys. 62, 453 (2013).

[45] D. M. Kennes, J. Lischner, and C. Karrasch, Strong correlations and $d+i d$ superconductivity in twisted bilayer graphene, Phys. Rev. B 98, 241407(R) (2018).

[46] L. Classen, C. Honerkamp, and M. M. Scherer, Competing phases of interacting electrons on triangular lattices in moiré heterostructures, Phys. Rev. B 99, 195120 (2019).

[47] C. Husemann and M. Salmhofer, Efficient parametrization of the vertex function, $\Omega$ scheme, and the $t, t^{\prime}$ Hubbard model at Van Hove filling, Phys. Rev. B 79, 195125 (2009).

[48] J. Lichtenstein, D. Sánchez de la Peña, D. Rohe, E. Di Napoli, C. Honerkamp, and S. Maier, High-performance functional Renormalization Group calculations for interacting fermions, Comput. Phys. Commun. 213, 100 (2017).

[49] C. J. Eckhardt, C. Honerkamp, K. Held, and A. Kauch, Truncated unity parquet solver, Phys. Rev. B 101, 155104 (2020).

[50] C. Hille, F. B. Kugler, C. J. Eckhardt, Y.-Y. He, A. Kauch, C. Honerkamp, A. Toschi, and S. Andergassen, Quantitative functional renormalization-group description of the two-dimensional Hubbard model, arXiv:2002.02733 [cond-mat.str-el].

[51] C. Honerkamp, Efficient vertex parametrization for the constrained functional renormalization group for effective 
low-energy interactions in multiband systems, Phys. Rev. B 98, 155132 (2018).

[52] C. Honerkamp, H. Shinaoka, F. F. Assaad, and P. Werner, Limitations of constrained random phase approximation downfolding, Phys. Rev. B 98, 235151 (2018).

[53] P. Moon and M. Koshino, Energy spectrum and quantum Hall effect in twisted bilayer graphene, Phys. Rev. B 85, 195458 (2012).

[54] K. Ohno, Some remarks on the Pariser-Parr-Pople method, Theoretica Chimica Acta 2, 219 (1964).

[55] T. O. Wehling, E. Şaşı̆̆̆lu, C. Friedrich, A. I. Lichtenstein, M. I. Katsnelson, and S. Blügel, Strength of Effective Coulomb Interactions in Graphene and Graphite, Phys. Rev. Lett. 106, 236805 (2011).

[56] M. Rösner, E. Şaşığlu, C. Friedrich, S. Blügel, and T. O. Wehling, Wannier function approach to realistic Coulomb interactions in layered materials and heterostructures, Phys. Rev. B 92, 085102 (2015).

[57] D. S. de la Peña, M. M. Scherer, and C. Honerkamp, Electronic instabilities of the AA-honeycomb bilayer, Ann. Phys. 526, 366 (2014).

[58] M. M. Scherer, S. Uebelacker, and C. Honerkamp, Instabilities of interacting electrons on the honeycomb bilayer, Phys. Rev. B 85, 235408 (2012).

[59] D. S. de la Peña, J. Lichtenstein, and C. Honerkamp, Competing electronic instabilities of extended Hubbard models on the honeycomb lattice: A functional renormalization group calculation with high-wave-vector resolution, Phys. Rev. B 95, 085143 (2017).

[60] Jülich Supercomputing Centre, JURECA: Modular supercomputer at Jülich Supercomputing Centre, J. Large-Scale Res. Facilities 4, A132 (2018). 